\title{
The Relationship between Internet Addiction and Temperament among Children and Adolescents
}

\author{
*Mohammad Q Abdullah \\ Department of Counseling Psychology, University of Aleppo, Syria
}

Submission: August 04, 2017; Published: August 22, 2017

*Corresponding author: Mohammad Q Abdullah, Department of Counseling Psychology, University of Aleppo, Syria; Email: mk.abdalah@yahoo.com

\begin{abstract}
This study examined the relationship between internet addiction and temperament among children and adolescents. Additionally, it aimed to explore the differences between boys and girls and between children and adolescents for internet addiction. 351 children (167 boys and 184 girls) aged from 10 to 15 years of age ( $M=11.6$ male, and 13.9 female), attending all primary and preparatory school located in Aleppo. The participants completed measures of Internet Addiction Scale for Children (IASC), and the Temperament Assessment Scale for Children (TASC). The findings showed that boys were more addicted than female. Results also revealed that positive and significant correlation between internet addiction and Intensity, Mood, Distractibility, and Physical Sensitivity, on one side, and negatively with Activity, and Regularity on the other side.

Keywords: Internet Addiction, Temperament, Children and Adolescents.

Abbreviations: PIU: Problematic Internet Use; IA: Internet Addiction; IAD: Internet Addiction Disorder; IA: Internet Abuse; IO: Internet Overuse; NS: Novelty Seeking; HA: Harm Avoidance; RD: Reward Dependence; PS: Persistence; SD: Self-Directedness; C: Cooperativeness; ST: Self-Transcendence; TASC: Temperament Assessment Scale for Children; IASC: Internet Addiction Scale for Children; FFM: Five Factor Model; BFFP: Big Five Factors of Personality.
\end{abstract}

\section{Introduction}

Since the 1990s, revolutionary technology has dramatically altered how information is successes, flooding the world with the internet, which has become a characteristic of our society Moreno et al. [1]. Several characteristics make the internet highly appealing such as easy availability, accessibility, affordability and anonymity, making it one of the most popular leisure-time activities among children and adolescents. The internet is a technology that facilitates accessing various kinds of information resources and information exchange easily via an expensive and safe way. Despite the positive consequences of communication via internet, overuse of internet may have several negative effects for children and adolescents Young [2].

The internet has become an integral part of children and young people's lives. The increased time spent online is prompting questions about whether they are in control of their internet usage. Concerns have been expressed that too much time on the internet can negatively influence several aspects of young people's lives, perhaps leading to: declining school results or even dropping out of school; increased family tension; abandoned hobbies; psychological problems such as depression, anxiety, or low self-esteem; and physical health problems due to sleep deprivation and lack of physical activity Smahel [3].

Problematic Internet use (PIU), Internet Addiction (IA), Internet Addiction Disorder (IAD), internet abuse, or Internet overuse (IO), refers to excessive internet usage, which causes negative consequences such as psychological, social occupational and academic difficulties Beard and Wolt [4]. Although a standardized definition of internet addiction has not been uniformly agreed upon, some researchers define the internet addiction as having les ability to control enthusiasm for internet activities, losing the importance of the time without being connected to the internet, extreme nervousness and aggressive behavior when deprived, and progressive deterioration in work and social and family functioning. Research on Internet Addiction continues to document severe effects of internet use, such as Depression and anxiety, academic failure, and social isolation Keltner and kring [5]. Several studies have underlined the unfavorable effects of internet addiction on physical and mental 
well-being, and most of children and adolescents with internet addiction were reported to have other psychiatric disorders. Mood disorders, hyperactivity and attention syndromes, disruptive behavior, anxiety, sleep and eating disorders are some proven internet addiction-related clinical situations Ko [6]. Excessive or over use of the Internet can be seen as a rewarding behavior, and through learning mechanisms, it may be used as an insufficient strategy to cope with some negative feelings $\mathrm{Du}$, Jiang and Vance [7].

Internet addiction is considered a disorder in need of further study, and the American Psychiatric Association has considered including the diagnosis of pathological computer use in the upcoming revision of the Diagnostic and Statistical Manual of mental Disorders ( $5^{\text {th }}$ edn), (DSM-V). Conversely, according to some researchers, appears hastily to consider internet addiction as discrete disease on a basis of DSM criteria, such as suffering and incapacity, as well as prognosis, temporal stability, and response to treatment Pies [8]. Overuse of internet related to a series of emotional and physical complication, and during the last 10 years in the psychological literature, the interplay between protective and risk indicators has been considered key in understanding developmental trajectories during childhood and adolescence. Researcher tried to identify risk and protective factors that influence addictive behavior, and they found that personality and familial factors influence addiction Pace [9].

Genetic factors are believed to account for about half of the likelihood that an individual will develop addiction, whereas environmental factors interact with the person's biology and affect the extent to which genetic factor exert their influence. Bargh and McKenna [10]. In this sense, protective factors that individual may acquire during early social interaction can moderate the extent to which genetic inclination lead to the development of an addiction. Recently, some authors have suggested a linkage between personality traits and various types of addiction, specifically, between temperament traits and addiction Lee and Jung [11]. There are numerous approaches to conceptualizing temperament, which can be defined as " biologically rooted individual differences in behavior tendencies that are present early in life and are relatively stable across various kinds of situations and aver the course of time», Pace [9]. Temperament can be defined as individual differences in reactivity, the responsively of the behavioral and psychological system, and self-regulation, as neural process that modulate reactivity is assumed to have constitutional basis.

The concept of temperament has gained prominence in recent years a persuasive and powerful framework for understanding individual differences among children. Described as the «how» of behavior, temperament refers to constitutional differences in the way that individual respond to their environments. These differences, which are conceptualized as stable across different situations and developmental periods, are believed to be based in aspects of physiology and neuroanatomy that underlie broad patterns of activity and response. Such differences, in interaction with the growing person's experience, are thought ultimately to play an important role in the development of personality, mental health, and adaptation to the social environment Rothbart Chew and Gartein [12]. Thomas and Chess's formulation, which sparked developments in the field in the last half century, postulated nine dimensions of temperament: Activity, Regularity, Adaptability, Approach/Withdrawal, Intensity, Mood, Persistence, Distractibility, and threshold Thomas and Chess [13]. Thomas and chess's formulation of temperament is distinctive in its origins in clinical researches with children's mental health.

Personality traits have been investigated using the Temperament and Characters Inventory (TCI), which is based on Cloninger's theory. The TCI is a battery of tests designed to assess differences between people based on seven basic dimensions of temperament and character. Cloninger's biopsychosocial model of personality includes the psychological dimensions of temperament and characters Cloninger [14]. Cloninger first concentrated on the four biogenic dimensions of temperament and later extended his model to include three dimensions of character. The four dimensions of temperament include: novelty seeking (NS), harm avoidance (HA), reward dependence (RD), and persistence (PS), and the three character dimentions include self-directedness (SD), cooperativeness (C), and selftranscendence (ST), Lee and Jung [15].

Generally, there are five characteristics describe an individual's temperament: emotional intensity, activity level, frustration tolerance, reaction to new people and reaction to change. The child's temperament is a key factor in understanding their behavior and the way they interact with others. There are three general types of temperaments often referred to as easygoing, slow-to-warm, and active/feisty. Temperament is not something a child or young chooses, nor it is something that a parent or caregiver created. A child's temperament shape the way he experiences the world.

Temperament traits of novelty or sensation seeking are reported to be significantly higher in substance users than in nonusers. Massc and tremplay [15]. In studies investigating the temperament features of adolescents with internet addiction were easily affected by feeling, emotionally less stable, imaginative, absorbed in thought, self-sufficient, experimenting, and preferred their own decision Yang [16]. Adolescents with internet addiction were shown to have higher scores on neuroticism and psychoticism temperament categories than those of the control group Cao and Su [17]. Temperament is believed to play an important role in children and adolescents, social and personality development, psychological adjustment, and different forms of addictions. For example, sensitivity could involve peripheral stimuli with emotional relevance, and an extensive body of research has linked effortful control to children's model task performance on tasks that assess 
the efficiency of the executive attention system Rothbart and Rueda [18]. The relationship between internet addiction and temperament has been assessed in few studies. For example, Oztuk reported that there is relation between internet addiction and affective temperament profiles, especially with anxious temperament.

Furthermore, emotional and behavioral problems are more frequent in adolescents who have problematic internet use. Children with internet addiction appeared high levels of avoidance, and scored lower in novelty seeking (tend to engage in high-risk and extreme sport) than non-addicted children Rutter [19].

Variability in average ratings of children's temperament in different cultures can be interpreted in three ways: First, it is possible that children's temperament really do vary across culture groups, for a variety of reasons including genetic and early environmental factors. Second, Parents in deferent cultures may have different expectations and values regarding children's temperament, which are reflected in their responses to the questionnaires, thus, particular kinds of child behavior may have dramatically different meaning in different cultures. Third, parental ethnotheories of child temperament may shape parenting practices in such a way as to influence the development and expression of child temperament over time; this may particularly true as the ethnotheories are shared with other important social groups and institutions Rothbart and Rueda [18].

The scientific study of ethnic in temperament began with Galton's contrast between the Chinese, the Americans, and the Africans. Since then, most of the research has been carried in Europe or the United States, where various groups have through a Eurocentric lens. Increasingly, however, scientists of nonEuropean ancestry have been adding to our store of knowledge and thereby broadening our perspective. By doing so, research on ethnic differences in temperament and behavior may help to promote mutual understanding and cooperation Rushton [20].

The relationship between internet addiction and temperament has been assessed in few researches. Cho et al. Underlined that problematic internet users reported lower novelty seeking than non-problematic internet users, suggesting that biogentic temperament patterns may be one of the etiologies of problematic internet overuse Pace [8]. Furthermore, the social control theory suggests that, when children and adolescents are close to their parents, they feel obligated to act in non-deviant to please their parents Wright and Cullen [21]; in contrast, a lack of support from parents can cause children to seek social support from the interacting experiences found in the virtual world Tichon and Shapiro [22].

The literatures showed that both parental practices and temperament are important predictors of children and adolescents adjustment, and their effects are additive, with each contributing a unique variance above the other Lengua [23]. Regarding the effect of gender on internet addiction, various studies have generated inconsistent results. Some studies indicated that internet addictive behavior was consistently high for male gender Ford and Miller [24], Bimber [25], while others found that females gender have spent more time online compared to males, Ono and Zavodny [26].

However, to our knowledge, there is not a study in the psychological literature addressing the correlation between temperament and internet addiction among children in our society. In the current study, we hypothesized that both temperament and family functioning of the children would be positively correlated with children's internet addiction. The second variable (family functioning) has been studied in our society Abdulla [27], while the first one (temperament) has been neglected.

The first aim of this study was to investigate the differences in internet addiction regarding tow variables: gender and developmental period, among a sample of Syrian children population. Second, the study aimed to investigate the relationship between temperament and internet addiction.

\section{Methods}

\section{Participants}

Participants in the study were 351 children (167 boys and 184 girls) aged from 10 to 15 years of age (M=11.6 male, and 13.9 female), attending all primary and preparatory school located in Aleppo.

\section{Measures}

Temperament: The Temperament Assessment Scale for Children (TASC) is a self-report instrument consisting of nine items: Activity (How much does the child wiggle and move around being red to, sitting at table, or playing alone), Regularity (Is the child regular about eating times, sleeping times, and bowel movement), First Reaction (How strong or violent are the child's reaction? Does the child laugh and cry energetically, or does he /she just smile and fuss mildly?), Adaptability (How quickly does the child adapt to changes in her /his schedule or routine), Intensity (How strong are the child's emotional reactions-negative or positive), Mood (The amount of happy, contented mood vs. cranky, serious analytical mood expressed by child), persistence (How long does the child continue with one activity? Does the child usually continue if it's difficult?), Distractibility (Is the child easily distracted, or do he / she ignore distractions? Will the child continue to work or play when other noises or children are present), and Physical Sensitivity (how aware is the child of slight noises, slight differences in temperature, differences in taste, and differences in clothing). The child could answer on a 5-point likert scale ranging from 1 to 5.The (TASC) is a highly reliable and internally consistent measure (Cornbrash $=.89$ ). 


\section{Psychology and Behavioral Science International Journal}

Internet Addiction: Internet Addiction scale for Children (IASC) is a self report instrument consisting of 30 items (e.g., " I feel that life without internet would be boring and empty», «I have attempted to spend less time on the internet but I have been unable to do so»). The (IASC) is highly reliable and internally consistent measure (Cronbach= .85).

\section{Data Analysis}

Statistical package for Social Sciences software (SPSS) was used for the analysis. Descriptive parameters were shown as mean, standard deviation or in percentage. Continuous variables were compared using the student $t$ test. Pearson's correlation tests were used to evaluate the relationship between the (TASC) and The (IASC).

\section{Results}

To explore gender differences in internet addiction, $t$ test has been used, and the results presented in Tables 1-3.

Table 1: Descriptive statistics of the differences for internet addiction between Boys and Girls.

\begin{tabular}{|c|c|c|c|c|c|c|}
\hline Variable & \multicolumn{2}{|c|}{ Boys } & \multicolumn{2}{c|}{ Girls } & t & Sig \\
\hline & SD & M & SD & M & & \\
\hline $\begin{array}{c}\text { Internet } \\
\text { addiction }\end{array}$ & 0.05 & 2.96 & 15.45 & 38.12 & 18.36 & 46.06 \\
\hline
\end{tabular}

Table 1 showed significant differences for internet addiction (sig $0.05)$. Of the sample, boys were found to be more addicted $(M=46.06)$ than girls $(M=38.12)$.

To examine the differences between children and adolescents for internet addiction, $\mathrm{t}$ test has been used, and the results presented in Table 2.

Table 2: Descriptive statistics of the differences for internet addiction between Children and Adolescents.

\begin{tabular}{|c|c|c|c|c|c|c|}
\hline Variable & \multicolumn{2}{|c|}{ CHILDREN } & \multicolumn{2}{c|}{ ADOLESCENTS } & t & Sig \\
\hline & M & SD & M & SD & & \\
\hline $\begin{array}{c}\text { Internet } \\
\text { addiction }\end{array}$ & 0.05 & 2.73 & 15.48 & 49.65 & 14.48 & 36.72 \\
\hline
\end{tabular}

Table 2 showed significant differences between children and adolescents for internet addiction. Of the sample, adolescents found to be more addicted $(M=49.65)$ than children $(M=36.72)$.

Table 2 revealed that positive and significant correlation between internet addiction and Intensity $(R=0.31$, Mood $(R=0.53)$, Distractibility $(R=0.51)$, and Physical sensitivity $(R=0.48)$, on one side, and negatively with Activity $(R=-0.36)$, and Regularity $(R=-0.27)$ on the other side.

Correlation analysis (Pearson's coefficient) has been used to investigate the relationship between temperament and internet addiction. The results presented in Table 3.

Table 3: The correlation coefficient between Internet addiction and temperament.

\begin{tabular}{|c|c|}
\hline Temperament/Internet Addiction & R \\
\hline Activity & $-0.36^{*}$ \\
\hline Regularity & -0.27 \\
\hline First Reaction & 0.23 \\
\hline
\end{tabular}

\begin{tabular}{|c|c|}
\hline Adaptability & -0.12 \\
\hline Intensity & $0.31^{*}$ \\
\hline Mood & $0.53^{*}$ \\
\hline Persistence & 0.28 \\
\hline Distractibility & $0.51^{*}$ \\
\hline Physical Sensitivity & $0.48^{*}$ \\
\hline
\end{tabular}

* Sig. 0.05

\section{Discussion}

In the present study, the prevalence of internet addiction was found among children. According to the findings, the prevalence of internet addiction was higher in boys than in girls. This finding is consistent with the results revealed in some studies such as Ha and Hwang [28] and Dufour [29]. There are some sex differences, which are relevant to internet use, such as social aggressiveness expressiveness and the structure of friendship Canary and Dindia [30]. There are also some differences between genders in certain areas of cognition, which can be effective on internet use Halpern [31]. In general, females are more likely to enjoy searching for information of personal interest other than the requirement of work Ford and Miller [24]. In addition, as part of social life, boys learn to hide their emotion, and girls learn to more freely express their emotions compared to boys. Ono and Zavodny [26] With respect to the outward expression of emotions, there are differences between genders Fischer [32].

It is considered that such differences between the two genders have in expression of emotions may be related to the distinct differences in their scores for internet addiction Oktan [33] on one side, and it is related to the temperament on the other side. The findings showed that, temperament was correlated significantly with, intensity, mood, distractibility and physical sensitivity and negatively with Activity and regularity. It is possible, therefore that children and adolescents with temperament predisposition to lack control over themselves and their activities were more vulnerable to developing addiction problems such internet overuse. In addition, it is interesting to note that other temperament dimensions, such as negative mood, sensitivity and distractibility many play role in the addicted behavior. According to the literature, the positive effect of the family affective involvement against internet salience confirm the important role that families functioning plays in the psychological development of their children Rushton [22].

The results also showed that internet addiction was found to be higher in adolescence than in childhood. This finding consistent with studies of Cao and $\mathrm{Su}$ [18] and Moreno [1]. According the relationship between temperament and activity, the mean internet addiction score was significantly higher in children who were not involved in any kind of physical activities. Similar result was found when frequency of children 
with internet addiction was compared between those with and without physical activity.

Frequency of internet addiction was significantly higher for children and adolescents who lacked any physical activity as compared to those who were in the habit doing physical activity Lengua [26]. Dependent users were found to rank highly in terms of self-reliance i.e., they did not feel a sense of alienation others feel when sitting alone, possibly because of the interactive functions of the internet), emotional sensitivity and reactivity (i.e., they are drawn to mental stimulation through endless databases and information available online), vigilance, low self-disclosure, and non-conformist characteristics (i.e., they may be drawn to the anonymity of the internet), Ross [34].

The personality refers to the all aspects of a person's individuality. Psychologists are aware of the variety in personal characteristics of internet users among children and adolescents, Mirin [35]. The temperament is an important determinant of personality, especially through the development in childhood and adolescence. One personality fruitful place to start in addressing the relationship between personality and internet usage is with Five Factor Model (FFM) or Big Five Factors of Personality (BFFP) is the broad classification of personality traits. The model separates the human personality into a series of five dimensional traits. The first trait, neuroticism, reflects emotional stability, and reflects a persona's tendency to experience psychological distress and high levels of trait are associated with sensitivity to danger. Extraversion, the second trait, reflects a tendency to be sociable and able to experience positive emotions, and represents an individual level of sociability and outgoingness Ross [34].

The third factor, openness to experience, represents an individual, a willingness to consider alternative approaches, be intellectually curious, and enjoy artistic pursuits. Agreeableness, the fourth factor, is another aspect of interpersonal behavior reflecting a tendency to be trusting, sympathetic and cooperative. The fifth dimension, conscientiousness reflects the degree to which an individual is organized, diligent, and scrupulous, careful, and shows self-control Muscanell [36].

Behavioral addictions demonstrate the core features of physical and psychological addictions such as mental rumination, mood variability, tolerance, withdrawal, interpersonal conflict and relapse Donovan [37]. According to the «self-mediation hypothesis», the patients usually use the substance to change their unwanted temperament status, to reduce their unbearable anxiety, and to cope with cognitive impairments Mirin [35] this can be seen in the internet addiction, which is also a behavioral addiction. Namely, repeating efforts to get online may be a way of decreasing the sensitivity of withdrawal symptoms such as anxiety. Additionally, the explanation of increased internet addiction frequency in children and adolescents with anxious temperament may be related to» self-mediation hypothesis» Samarein [38].
Children high in harm avoidance tend to be cautious, careful, fearful, and tense. Ha, Hwang [28] have reported that high harm avoidance, low self-defectiveness, low cooperativeness and high self-transcendence were correlated with internet addiction. Additionally, those engaging with a range of risk factors reported higher prevalence of problem behavior than others did. Youth who suffered from emotional problems or high levels of psychological distress spent the most time online or playing games.

\section{Conclusion}

The excessive use of internet becomes widespread and it certainly helps people to reach information to communicate with each other across boundaries and it provides many opportunities. However, it is also a tool, which can deprive individuals from face-to-face interaction and benefits stem from sharing the same physical environment, especially through the development of child and adolescent. The findings showed that boys more addicted than girls did, also, adolescents more addicted than children did. Additionally, the internet over usage correlated positively with intensity, mood, distractibility and physical sensitivity, and negatively with Activity and regularity.

Based on the findings, it is concluded that excessive internet use clearly represents a health risk for a significant portion of the population of children and adolescents. Excessive time spent in front of a computer and web overuse may detrimental to virous aspects of their lives including the social, functional, physical and psychological aspects, forsaking other important priorities in the process, at risk for a range of morbidities and, in extreme, even mortality Samarein [38].

Internet addiction specifically refers to the following feelings and attitudes: the preference of the virtual life over real life, and the use of internet to escape from negative emotions, and thought. Especially during childhood and adolescence, the excessive use of internet can easily lead to compulsive if some predisposing personal factors are present, such as temperament (e.g., negative mood, low level of activity) [39].

\section{Recommendations}

The results of the current study have important implications for children and adolescents counseling programs. Particularly, this study provides support for the strong focus on temperament and personality traits involvement that is an essential tenet of the systems of care approach. The constraint of the study is that the sample is comprised of children and adolescents. The study may be repeated with different populations and through the developmental stages of life span. The existence of negative relationship between activity, regularity and internet addiction has been determined; the predictive value of negative/positive mood for predicting internet addiction degree has been implicated. In the future researches, psychological and social factors may be explored for aspects of their influence on the relationship between internet addiction and temperament / 
personality traits. We recommend to further studies focusing on the casual connection between these variables, and using comparative and longitudinal research methods through life sp an.

\section{Acknowledgment}

The researcher thank to teachers and psychological counselors who supervised and administered the implication of the measures in the schools, and to the children who participated in the study.

\section{References}

1. Moreno MA, Jenchick L, Cox E, Young E, Christakis D (2011) Problematic internet use among US youth: A systematic review. Archives Pediatrics and Adolescent medicine 165: 797-805.

2. Young KS (2009) Internet addiction: Diagnosis and treatment consideration. Journal Contemporary Psychotherapy 39: 241-246.

3. Smahel D, Helper E, Green L, Kalmus V, Blinka L, et al. (2012) Excessive internet use among European Children. EU Kids Online: 1-10.

4. Beard KW, Wolf EM (2001) Modification in the proposed diagnostic criteria for internet addiction. Cyber Psychology and Behavior 4: 377 383

5. Keltner D, Kring A (1998) Emotion, social function and psychopathology. Review of General Psychology 2: 320-342.

6. Ko C, Yen J, Chen C (2012) The association between internet addiction and psychiatric disorders: A review of the literature. European Psychiatry 27(1): 1-8.

7. Du Y, Jiang W, Vance A (2010) Longer term effects of randomized, controlled group cognitive behavioral therapy for internet addiction in adolescent students in Shanghai. Australian and NewZel and Journal of Psychiatry 44(2): 129-134.

8. Pies R (2009) Should DSM-V designate "internet addiction" a mental disorder. Psychiatry 6: 31-37.

9. Pace U, Zappulla C, Guzzo G, Maggio R, Laudani C (2014) Internet assertion, temperament and the moderator role of family emotional involvement. International Journal of mental Health and addiction 12(1): 52-63.

10. Bargh J and Mc Kenna K (2004) The internet and social life. Annual review of Psychology 55: 573-590.

11. Lee M, Jung L (2012) Comparisons of temperament and character between problematic internet users and problematic drug users in Korean adolescents. Open Journal of Psychiatry 2: 228-234.

12. Rothbart M, Chew K, Gartein M (2001) Assessment of temperament in early development. In: LT Singer, PS Zeskind (Eds.). Biobehavioral assessment of the infant. Guilford Press, New York, USA. pp 190-208.

13. Thomas A, Chess S (1977) Temperament and development. Brunner/ Mazel, New York, USA.

14. Cloninger C, Przbeck T, Svrakic D, Wetzel R (1994) The temperament and characters inventory: A guide to its development and use. Center for Psychobiology of personality. Washinghton University, St. Louis, USA.

15. Massc L, Tremplay R (1997) Behavior of boys in kindergarten and the onset of substance use during adolescence. Archives of General Psychiatry 54(1): 62-68.

16. Yang C, Choe M, Baity J, Lee J, Choe S (2005) Psychiatric syndromes in adolescents with internet addiction: comparison with substance use. Psychiatry and Clinical Neurosciences 62(1): 9-16.
17. Cao E, Su L (2007) Internet addiction among Chinese adolescents. Child. Care, Health and development 33(3): 275-281.

18. Rothbart M, Rueda M (2005) The development of effortful control. In U Mayr, E Awh, S Keele (Eds). Developing individuality in the human brain: A tribute to Michael I posner, American Psychological Association, Washington, USA. pp: 167-188.

19. Rutter M (2007) Psychopathological development across adolescence. Journal of Youth Adolescents 36: 101-110.

20. Rushton P (1999) Ethnic differences in temperament. In: Y Lee, C Mc Cauley, J Draguns (Eds.). Personality and person perception across cultures Mahwath, Erlbaum, New Jersey, USA pp 45-63.

21. Wright JP, Cullen FT (2004) Parental efficacy and delinquent behavior: do control and support matter? Criminology 39: 677-604.

22. Tichon JG, Shapiro M (2003) The process of shaping social support in cyberspace. Cyber Psychology and Behavior 6(2): 161-170.

23. Lengua L (2006) Growth in temperament and parenting as predictors of adjustment during children's transition to adolescence. Developmental Psychology 42(5): 819-832.

24. Ford N, Miller D (2002) Gender differences in internet perceptions and use. Aslib Proceeding 4: 183-192.

25. Bimber B (2000) Measuring the gender gap on the internet. Social Science Quarterly 81(3): 868-876.

26. Ono H, Zavodny M (2003) Gender and the internet. Social science quarterly 84(1): 111-121.

27. Abdullah QM (1992) The family problems of youth: Interaction of socialization and psychological health. Acta Paedopsychiatrica 55(4): 217-220.

28. Ha Y, Hwang W (2014) Gender differences in internet addiction associated with psychological health indicators among adolescents. International Journal of Mental Health and Addiction 12(5): 660-669.

29. Dufour M, Brunelle N, Tremblay J, Cousineau M, Khazaal Y, et al. (2016) Gender differences in internet use and internet problems among Quebec High school students. The Canadian Journal of Psychiatry, 61(10): 663-668.

30. Canary D, Dindia K (1998) Sex differences and similarities in communication. Mahwah N J Lawrence Erlbaum.

31. Halpern D (1996) Changing data, changing mind: What the data on cognitive sex differences tell us and what we hear. Learning and individual differences 8(1): 73-82.

32. Fischer A, Mosquera P, Van Vianen A, Manstead A (2004) Gender and culture differences in emotion. Emotion 4(1): 87-94.

33. Oktann V (2011) The predictive relationship between emotion management skills and internet addiction. Social Behavior and Personality 39(10): 1425-1430.

34. Ross C, Orr E, Sisic M, Arseneault J, Simmerin M (2009) Personality and motivations associated with Facebook use. Computers in Human Behavior 25: 578-586.

35. Mirin S, Weiss D, Micheal J, Griffin M (2012) Psychopathology in substance abusers: diagnosis and treatment. American Journal of drug and Alcohol abuse 14(2): 139-157.

36. Muscanell N, Gadagno R (2011) Make new friends or keep the old: Gender and personality differences in social networking use. Computers in Human Behavior 28: 107-112.

37. Donovan J (2004) Adolescent alcohol initiation: A review of psychological risk factors. Journal of Adolescents Mental Health 35(6): 7-18. 
38. Samarein Z, Far N, Yekleh M, Tamasebi S, Ramezani Y, et al. (2013) Relationship between personality traits and internet addiction of students at Kharazmi University. International Journal of Psychology and behavioral research 2(1): 10-17.
39. Abdullah QM (2014) Internet addiction and its relationship with mental health among children. Journal of Arab Childhood 64: 9-31.

Your next submission with Juniper Publishers will reach you the below assets

- Quality Editorial service

- Swift Peer Review

- Reprints availability

- E-prints Service

- Manuscript Podcast for convenient understanding

- Global attainment for your research

- Manuscript accessibility in different formats

( Pdf, E-pub, Full Text, Audio)

- Unceasing customer service

Track the below URL for one-step submission https://juniperpublishers.com/online-submission.php 